\title{
Fonction sexuelle et bilan sexologique de l'homme paraplégique et tétraplégique
}

\author{
${ }^{*}$ F. COURTOIS, ${ }^{* * K}$. CHARVIER, ${ }^{* *}$ A. LERICHE \\ * Institut de réadaptation de Montréal, Montréal, H3S 2J4 Canada \\ ** Hôpital Henry Gabriel, 69230 Lyon, France.
}

\section{RESUME}

Cet article décrit l'examen sexologique du blessé médullaire. Le bilan complet comprend une entrevue clinique, un examen neurologique, un EMG du plancher pelvien, un bilan urodynamique, une manométrie ano-rectale et un enregistrement physiologique de la réponse sexuelle en situation réflexe et psychogène. En l'absence d'un médecin, d'autres professionnels de la réadaptation ou le psychothérapeute et sexothérapeute peuvent procéder à un interrogatoire clinique plus élaboré qui évalue nombre des composantes de l'examen physique. Les résultats du bilan permettent de cerner le niveau de lésion par rapport à l'innervation génitale et ainsi de procéder à un pronostic de la fonction érectile et éjaculatoire résiduelle. L'enregistrement physiologique permet par ailleurs de confirmer ou infirmer le diagnostic initial et d'explorer la possibilité de dysfonction fortement psychogène, par opposition à une dysfonction strictement organique chez l'homme blessé médullaire.

Mots clés : érection, éjaculation, lésion médullaire, paraplégie.

Remerciements : Les projets d'etudes qui ont mené à cet article de synthèse ont bénéficié de subventions du Fonds de la Recherche en Santé du Québec (F.R.S.Q.) et du Fonds pour les chercheurs et l'Aide à la Recherche (F.C.A.R.) du gouvernement du Québec.

\section{INTRODUCTION}

Les blessures médullaires occasionnent des dysfonctions sexuelles directement reliées au handicap physique puisque ce sont des affections du système nerveux et que celuici est directement impliqué dans la fonction érectile et éjaculatoire. Dans une série d'études sur la fonction sexuelle de l'homme blessé médullaire $[3,4]$ nous avons néanmoins démontré que peu de lésions entrainaient d'impuissances véritables. Chaque lésion est plutôt associée à un mode de stimulation optimal, réflexe dans un cas, psychogène dans l'autre.

La consultation sexologique du blessé médullaire devrait donc établir le mode de fonctionnement résiduel du patient. En général, aucun diagnostic n'est fait puisque la dysfonction est associée au facteur organique indéniable. Les traitements sont donc offerts en première entrevue et varient entre les auto injections d'agents vasoactifs, comme la papavérine $[1,5]$ et la prostaglandine [12], les systèmes de vacuum [8, 13,15 ] et l'implant chirurgical de prothèses péniennes $[7,9]$. Ces traitements sont très efficaces pour induire des érections mécaniques et sont certainement indiqués pour cette population clinique. Néanmoins, près de la moitié des hommes paraplégiques délaissent les traitements populaires d'injections pharmacologiques pour combler leurs difficultés sexuelles $[1,5]$, et nos données préliminaires montrent que l'effet des traitements pharmacologiques n'est pas 
entièrement indépendant de la capacité érectile résiduelle du sujet. Il est donc souhaitable de discerner le niveau de lésion par rapport à l'innervation génitale pour identifier le mode de stimulation qui sera apte à provoquer des érections chez l'homme paraplégique et tétraplégique.

La Figure 1 montre l'innervation de l'appareil génital qui comporte deux voies nerveuses, une voie sacrée responsable de l'érection réflexe et une voie dorso-lombaire responsable de l'érection psychogène. La voie sacrée est activée par une stimulation directe des organes génitaux telle qu'elle est pratiquée durant la masturbation ou les attouchements. Cette stimulation donne naissance à un influx nerveux qui parcourt les fibres sensitives du nerf honteux interne, connues sous le nom de nerf dorsal de la verge (NDV), et qui se rendent aux métamères $\mathrm{S} 2, \mathrm{~S} 3, \mathrm{~S} 4[6]$. De ces métamères, des synapses sont établies avec les fibres motrices du nerf honteux interne [6], responsables respectivement de la tumescence et de la rigidité pénienne.

La voie dorso-lombaire implique un vaste choix de stimulations non-génitales incluant les fantasmes, les stimulations visuelles, auditives, olfactives ou même somesthésiques lorsque non-génitales. Ces stimulations sont intégrées dans les centres supérieurs (cortex, hypothalamus) puis transmises aux métamères D11, D12, L, F2 . De ces métamères, des synapses sont établies avec les fibres orthosympathiques préganglionnaires, connues sous le nom de nerfs splanchniques (NS), qui font synapse dans les ganglions coeliaque (GC), mésentérique supérieur (GMS) et mésentérique inférieur (GMI) avec les fibres postganglionnaires du nerf hypogastrique (NH) [6].

Etant donné la double innervation de l'appareil génital, différents cas de figures peuvent se présenter à la suite d'une lésion médullaire. Plutôt que de juger de la lésion en termes absolus (ex : C6, D11, L5), il est donc plus aisé en sexologie de juger de la lésion en fonction de l'innervation génitale.
La Figure 2 montre qu'une lésion peut se situer (A) au-dessus de la double innervation génitale, (C) au niveau exclusif de l'innervation sacrée (lésion du cône médullaire) et (D) à un niveau étendu de l'innervation sacrée (lésion de la queue de cheval). Chaque cas de figure est associé à une capacité érectile et éjaculatoire différente, ces capacités ayant été démontrées dans nos études expérimentales $[3,4]$ ou vérifiées par notre expérience clinique.

\section{LESION AU DESSSUS DE LA DOUBLE INNERVATION GENITALE}

\section{Capacité érectile}

La coupe A de la Figure 2 montre qu'une lésion cervicale ou une lésion dorsale de D1 à $\mathrm{D} 10$ se situe au-dessus de l'innervation sacrée et dorso-lombaire. Ces lésions n'entravent pas le fonctionnement du circuit sacré-réflexe si bien que l'individu peut maintenir des érections à la suite d'une stimulation directe des organes génitaux, c'est-à -dire en réponse à la masturbation, aux attouchements ou la pénétration.

Lorsque la lésion est complète, il y a interruption des connexions entre la moelle et les centres supérieurs si bien que la réponse à la stimulation psychogène est perdue. L'individu garde néanmoins la réponse psychologique du désir et du plaisir, mais perd l'effet de la stimulation psychogène sur les organes génitaux.

\section{Capacité éjaculatoire}

Les individus blessés au-dessus de la double innervation génitale peuvent garder l'ensemble du processus éjaculatoire puisque l'innervation sacrée et dorso-lombaire impliquée dans l'émission de sperme (sécrétion des organes reproducteurs internes dans l'urêtre proximal) et l'éjaculation (expulsion en jet du sperme hors du méat urétral) est intact.

En réalité, toutefois, on observe peu d'éjaculations naturelles chez l'homme tétraplégique ou paraplégique haut. Ces hommes 


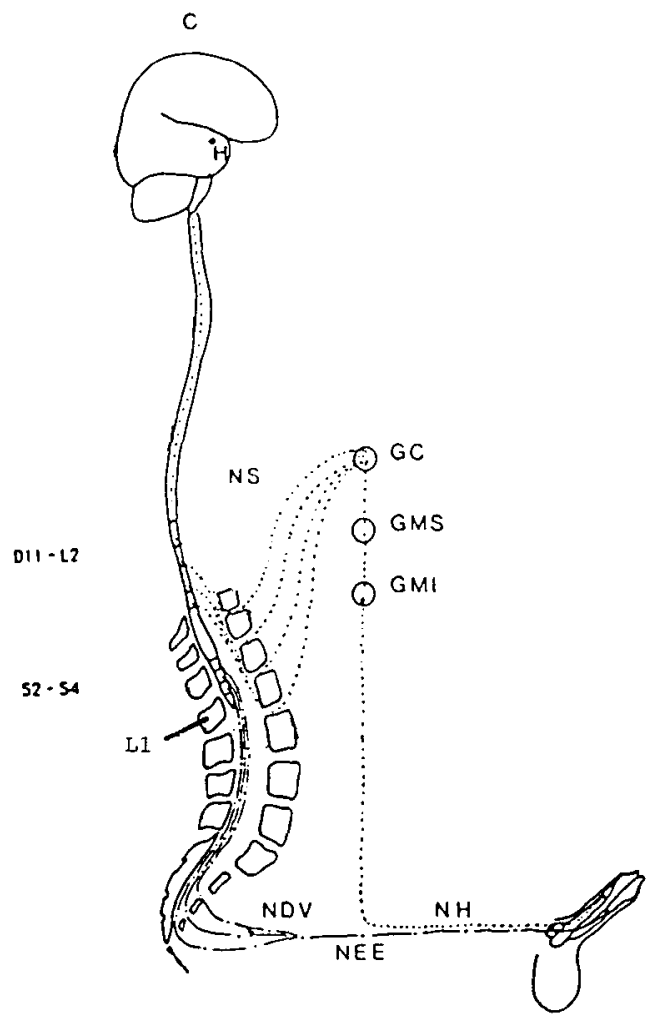

INNERVATION DE L'APPAREIL GENITAL
Figure 1 : Innervation de l'appareil génital de l'homme, avec le circuit sacré (S2-S4) responsable de l'érection réflexe et le circuit dorso-lombaire (D11-L12) responsable de l'érection psychogène. Les abréviations se lisent comme suit : C, Cortex ; GC, Ganglion coeliaque ; GMS, Ganglion mésentérique supérieur; GMI, Ganglion mésentérique inférieur ; NDV, Nerf dorsal de la verge ; NEE, Nerf érecteur d'Eckard ; NH, Nerf Honteux interne; NS, Nerfs splanchniques.

Figure 2 : Effet de lésions médullaires sur la fonction sexuelle. Partie A. Lésion cervicale ou dorsale se situant au-dessus de la double innervation génitale. Partie $B$. Lésion lombaire se situant entre les deux systèmes. Partie C. Lésion de circuit sacré limitée au cône médullaire. Partie $D$. Lésion du circuit sacré étendue à la queue de cheval.
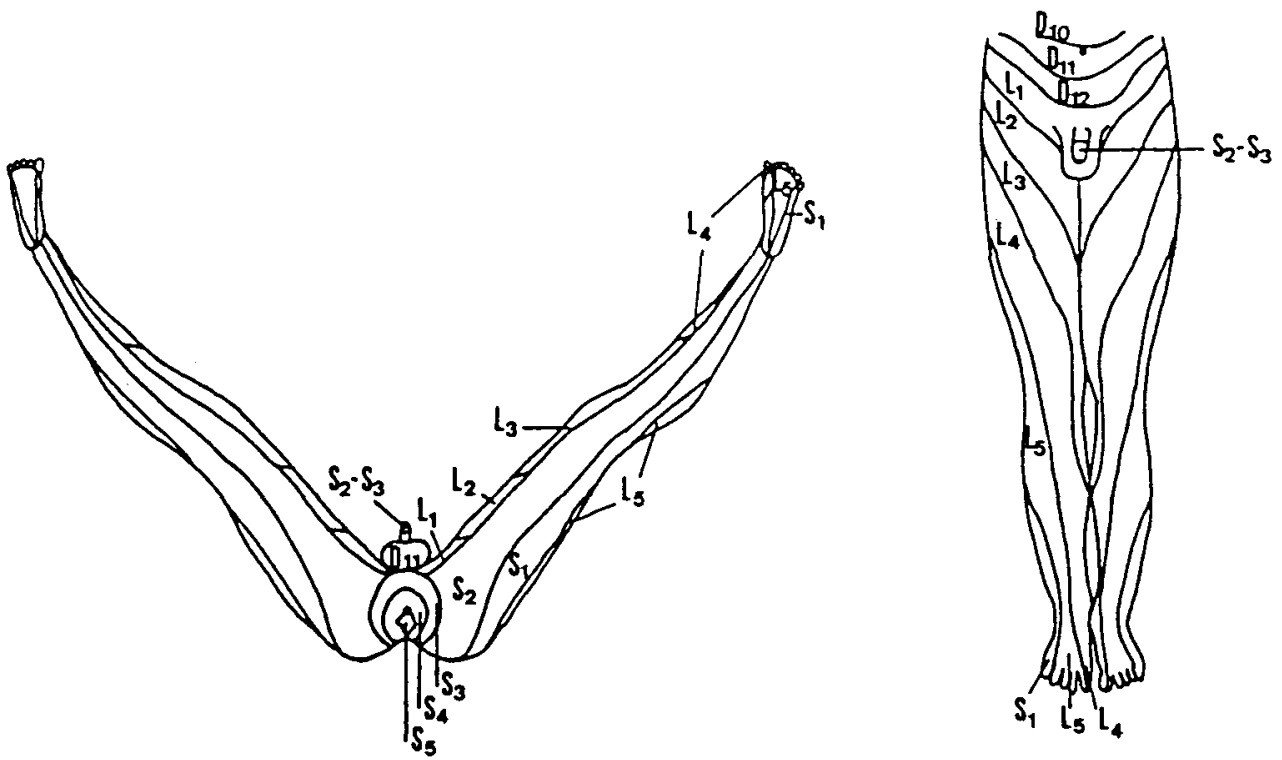

EXPLORATION DES VOIES SENSITIVES 
ayant perdu leur sensibilité, ne peuvent plus ajuster la notion du corps pour maximiser la stimulation et atteindre le seuil de déclenchement du processus éjaculatoire. Dans certains cas, l'individu apprend à tirer profit de sa spasticité pour déclencher l'éjaculation en stade final d'excitation sexuelle. Ces individus ont généralement des lésions hautes - au-dessus de D6 - et montrent des phénomènes de dysréflexie associés à des équivalents d'orgasmes.

Lorsque l'éjaculation n'apparait pas naturellement elle reste possible bien que provoquée artificiellement. L'activité réflexe étant préservée, et les conditions médullaires entre les deux circuits étant maintenues, la stimulation périnétale peut entrainer l'éjaculation. Le vibromasseur est ainsi utilisé en stimulation tonique sur la couronne du gland pour provoquer des éjaculations, et ce même vibromasseur peut être combiné à un agent pharmacologique (ex: Physostigmine, Guthron) pour augmenter les chances de réussite (il augmente néanmoins les effets secondaires de nausées et de céphalées).

L'électrostimulation rectale est également utilisée pour provoquer des éjaculations, et elle est probablement des plus efficaces, bien qu'elle ne puisse être utilisée que sur des lésions complètes puisque douloureuse. Cette stimulation active non seulement le circuit sensitif sacré (qui peut donc transmettre l'influx sensitif au centre dorso-lombaire) mais stimule également les organes reproducteurs internes de façon plus directe.

Le déclenchement du processus éjaculatoire n'assure pourtant pas la fertilité, ni même la présence de sperme. Dans ce dernier cas, les signes d'éjaculation peuvent avoir été présents (contractions rythmiques des testicules, dysréflexie autonome) sans qu'il y ait eu recueil de sperme. La présence de spermatozoïdes est alors recherchée dans l'urine, qui est recueillie lors de la miction suivant l'éjaculation. Inversement, il arrive que les blessés médullaires rapportent des épisodes de dysréflexie pendant la nuit, sans en comprendre la raison. Il est alors utile d'effectuer une analyse d'urine pour vérifier s'il y a eu éjaculation spontanée déclencheur du phénomène dysréflexique.

\section{LESION DU CONE MEDULLAIRE}

\section{Capacité érectile.}

La partie $\mathrm{C}$ de la Figure 2 montre une lésion limitée à la région sacrée de la moelle épinière, connue sous le nom de lésion du cône médullaire. Puisque le circuit sacréréflexe de l'érection est endommagé, la réponse érectile suite à une stimulation directe des organes génitaux est perdue. Cependant, le patient peut maintenir des érections par le biais de la stimulation psychogène puisque les centres supérieurs restent connectés au centre dorso-lombaire [3, 4]. Contrairement à l'individu dont la lésion se situe au-dessus de la double innervation génitale, l'homme paraplégique du cône médullaire maintiendra et l'excitation psychologique et l'effet psychogène de la stimulation sur la réponse pénienne.

\section{Capacité éjaculatoire}

Etant donné l'activation du système dorsolombaire pendant l'érection psychogène, les individus blessés au niveau du cône médullaire présentent des émissions de sperme associées à l'érection [3]. En fait, ces patients se plaignent souvent de troubles d'éjaculation précoce qui, dans ce cas, sont d'origine organique. Le motif initial de consultation est donc un problème d'érection qui sera reclassifié comme trouble d'éjaculation.

Par ailleurs, ces individus dont le cône médullaire est endommagé ont perdu l'activité du circuit sacré et ont donc perdu la contraction des muscle bulbocaverneux et ischiocaverneux. Le processus éjaculatoire ne peut donc avoir lieu en jet, et l'émission de sperme apparaitra par écoulement, phénomène que les auteurs par le passé ont appelé "éjaculation baveuse“. Il est néanmoins intéressant de noter que tous les 
individus (100\%) de nos études [3] dont la lésion se trouvait limitée au cône médullaire présentaient de telles éjaculations baveuses. La classification précise des lésions a donc un impact clinique certain qui justifie l'application d'un diagnostic chez le blessé médullaire.

\section{LESION DE LA QUEUE DE CHEVAL}

\section{Capacité érectile}

La partie D de la Figure 2 montre une lésion de la queue de cheval qui interrompt l'activité réflexe du centre sacré mais qui pourrait permettre l'activation du centre dorso-lombaire par le biais de la stimulation psychogène. En réalité, toutefois, les lésions de la queue de cheval représentent une entité neurologique qui mériterait d'être reclassifiée en plus petits éléments. Ainsi, une lésion à la limite supérieure de la queue de cheval peut endommager jusqu'aux métamères D12-L1. Les motoneurones inférieurs étant lésés, cette atteinte peut entrainer une perte complète de l'érection réflexe et psychogène. Une atteinte moyenne ou basse de la queue de cheval peut, en contrepartie, permettre l'activité psychogène lorsque l'activité érectile réflexe est perdue.

Malgré ces possibilités, le portrait clinique des lésions de la queue de cheval se complique du fait qu'elle ne sont que très rarement complètes. Le fonctionnement sexuel résiduel ne pourra donc être établi que par des explorations plus sophistiquées -EMG du plancher pelvien, bilan uro-dynamique, manométrie ano-rectale- qui cerneront l'état fonctionnel de chacun des circuits de l'érection et qui détermineront le portrait clinique du patient.

\section{Capacité éjaculatoire}

Toute comme la capacité érectile, la capacité éjaculatoire de l'homme dont la blessure atteint la queue de cheval est difficile à déterminer. Contrairement au sujet dont la lésion se situe au cône médullaire, et qui montre une émission par écoulement en présence d'érection psychogène, une atteinte de la queue de cheval peut préserver l'érection psychogène sans pour autant entrainer d'émission de sperme. Ces résultats, tout en restant partiellement inexpliqués, semblent cohérents avec certaines études animales qui montrent que l'érection provoquée par stimulation génitale inhibe le processus éjaculatoire. La capacité reproductive naturelle qui caractérise les lésions du cône médullaire n'est donc pas aussi clairement établie pour les lésions de la queue de cheval. Un bilan plus complet devra encore une fois être effectué pour vérifier si l'atteinte partielle permet une certaine activité sacrée. Dns ce cas le vibromasseur avec ou sans injections pharmacologiques pourra entrainer l'émission. Inversement, si l'atteinte permet une activité strictement dorso-lombaire, l'électostimulation rectale sera plus appropriée parce que stimulant directement les organes reproducteurs internes.

\section{LESION ENTRE LES DEUX SYSTEMES}

\section{Capacité érectile}

Les lésions entre les deux systèmes sont illustrées sur la partie B de la Figure 2. Puisqu'elles préservent le centre médullaire sacré et qu'elles préservent les connexions entre les centres supérieurs et le circuit dorso-lombaire, ces lésions permettent l'érection par stimulation réflexe ainsi que par stimulation psychogène. Néanmoins, l'effet additif de l'excitation psychogène et de la stimulation réflexe ne pourra se faire au niveau médullaire.

\section{Capacité éjaculatoire}

Théoriquement, l'émission devrait être assurée par la stimulation psychogène dans ce groupe puisque le circuit dorso-lombaire reste connecté aux centres supérieurs, par opposition au circuit sacré. En pratique, nous avons trouvé des émissions naturelles chez $40 \%$ de ces sujets [3].

Il est peu probable que le vibromasseur, qui requiert la transmission de l'influx sensitif 


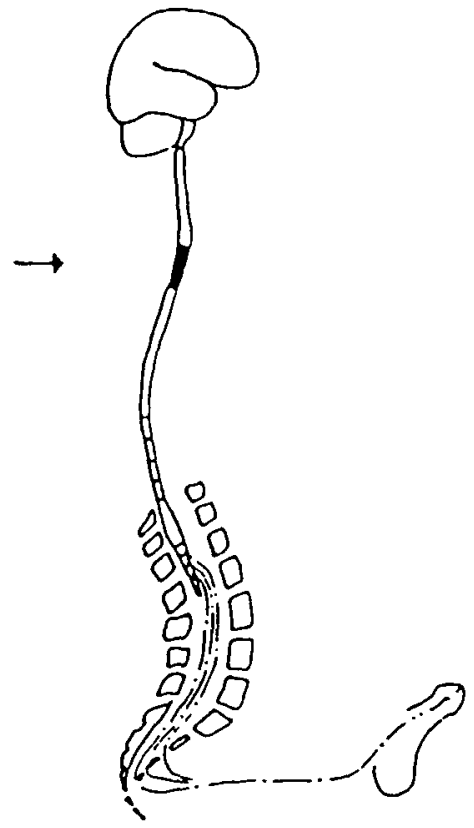

A - LESION CERVICALE OU DORSALE

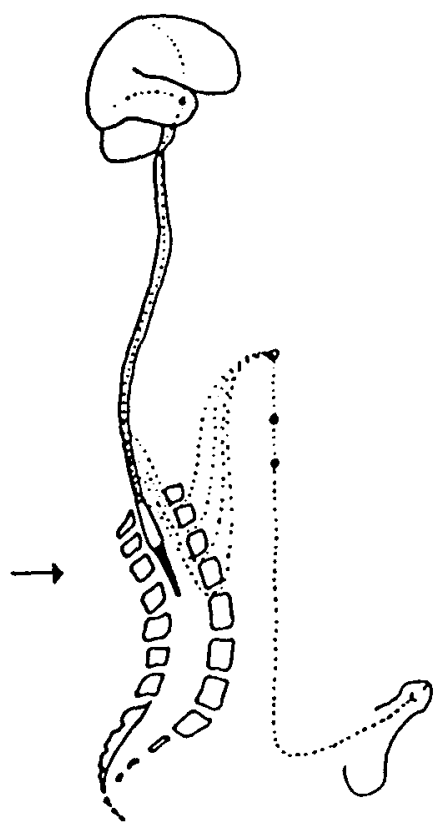

C - LESION DU CONE MEDULLAIRE

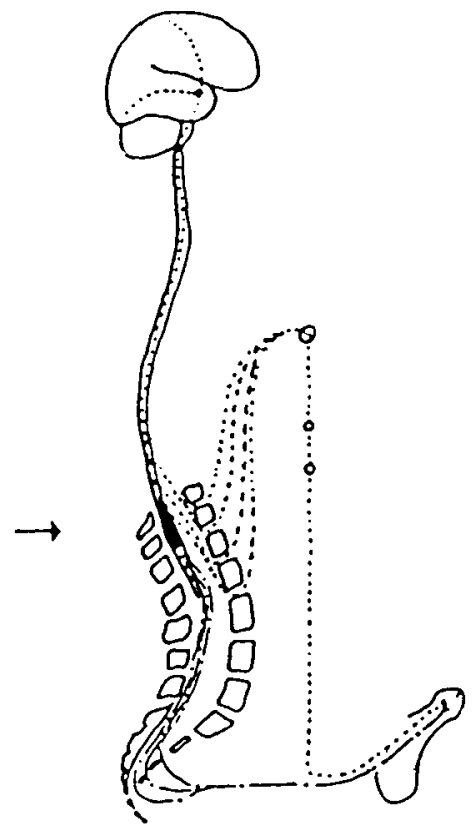

B - LESION LOMBAIRE

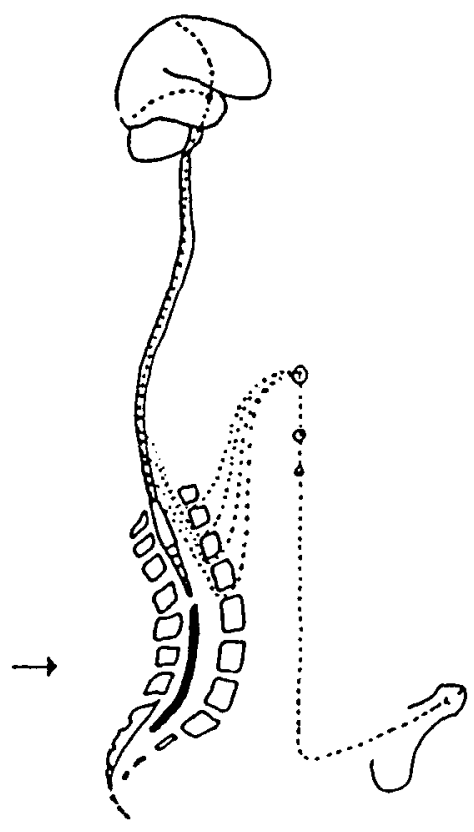

D - LESION DE LA QUEUE DE CHEVAL

Figure 3 : Schéma des dermatomes de la région périnéale tels qu'ils sont utilisés lors du bilan sexologique. 
du circuit sacré au circuit dorso-lombaire, donne des résultats satisfaisants. En contre partie, l'électrostimulation rectale devrait être le moyen le plus efficace pour recueillir le sperme puisqu'elle stimule plus directement les organes reproducteurs internes.

\section{EVALUATION DE LA FONCTION SEXUELLE RESIDUELLE}

L'ensemble de ces données cliniques montre qu'il est essentiel d'effectuer un examen sexologique du blessé médullaire pour déterminer le type de lésion qui nous permettra de prédire la capacité sexuelle du sujet. L'examen sexologique devrait, idéalement, être suivi d'un enregistrement physiologique de la réponse sexuelle en situation réflexe et psychogène pour objectiver le diagnostic initial, ou pour émettre un diagnostic différentiel entre un atteinte principalement organique et une atteinte principalement psychogène.

Le bilan complet comprend un examen neurologique, un EMG du plancher pelvien, un bilan uro-dynamique et une manométrie ano-rectale. En l'absence d'un médecin, le professionnel en réadaptation ou le psychologue-sexologue peut, néanmoins, procéder à l'interrogatoire clinique pour estimer nombre des composantes de cet examen.

\section{METHODE}

\section{Examen neurologique}

Tel que mentionné plus haut, l'examen du niveau de lésion ne se fait pas en termes absolus mais pour vérifier le niveau de la lésion par rapport à l'innervation génitale. L'examen neurologique évalue la présence de réflexes périnaux, la présence de commande volontaire du plancher pelvien et la sensibilité dermique pelvienne.

\section{Examen des réflexes périnaux et commandes volontaires du plancher pelvien}

Les réflexes périnaux explorent l'état de l'innervation sacrée. Tel qu'illustré au tableau 1, l'examen comprend le réflexe anal externe (S5-S4), le cutané-anal (S4), le bulbocaverneux (S4-S3) et le balano-rectal ((S3) [14]. Les réflexes anal externe et cutané-anal impliquent une stimulation de la marge anale, via le toucher rectal ( $R$. anal externe) ou via une stimulation algique ( $R$. cutané-anal), et entraine une contraction sphinctérienne sentie au toucher ( $R$. anal externe), notée visuellement (R. cutanéanal) ou enregistrée en électromyographie (EMG) [10]. Les réflexes bulbocaverneux et balano-anal impliquent une stimulation mécanique du gland (par pincement) qui entraine une contraction du muscle bulbocaverneux et du sphincter anal [10].

\section{Examen de la sensibilité dermique}

L'exploration des dermatomes se limite aux régions $\mathrm{S} 5$ à $\mathrm{D} 10$ puisque toute atteinte audessus de D10 représente une lésion au dessus de la double innervation de l'appareil génital. La Figure 2 illustre les dermatomes d'intérêt et montre que la sensibilité de la région périnale résulte de l'innervation $\mathrm{S} 5$, $\mathrm{S} 4$ et $\mathrm{S} 3$, que celle des deux-tiers distaux de la verge résulte de l'innervation S3 et S2, celle de la fesse résulte de l'innervation S2 et $\mathrm{S} 1$, celle de la face postérieure et latérale de la jambe résulte de l'innervation $\mathrm{S} 2, \mathrm{~S} 1$ et L5 et celle du petit orteil résulte de l'innervation de S1 [14].

Les dermatomes qui recoupent l'innervation dorso-lombaire comprennent la face interne de la cuisse en $\mathrm{L} 2$ et $\mathrm{L} 1$, de l'aine en $\mathrm{L} 1$, du tiers proximal de la verge en $L 1$, de la région abdominale inférieure en D12 et D11 et de la sensation de pression du testicule en D11.

Les tests de sensibilité permettent donc de situer le niveau de la lésion en fonction des dermatomes. L'exploration des divers types de sensibilité (tactile, algique, thermique) permet en outre de vérifier l'étendue de la lésion. En effet, la sensibilité tactile permet d'évaluer l'état des cordons postérieurs, alors que la sensibilité algique et thermique évalue l'état des cordons antéro-latéraux. 
Tableau 1 : Exploration des réflexes périnéaux et voie motrices.

\section{REFLEXE ANAL EXTERNE (S4-S5)}

Stimulation du sphincter anal durant le toucher rectal et contraction du sphincter sentie au toucher.

\section{REFLEXE CUTANE-ANAL (S4-S5)}

Stimulation algique (à l'aiguille) de la marge anale et contraction du sphincter notée visuellement.

\section{REFLEXE BULBOCAVERNEUX (S3-S4)}

Stimulation du gland par pincement et contraction du sphincter anal sentie au toucher rectal.

\section{COMMANDE VOLONTAIRE DU SPHINCTER ANAL}

Demander au patient de serrer les fesses et noter la contraction du sphincter au toucher anal.

\section{COMMANDE VOLONTAIRE DU MUSCULE BULBOCAVERNEUX}

Demander au patient de serrer les fesses et noter la contraction du muscle bulbocaverneux.

Puisque les faisceaux antéro-latéraux sont également associés à la transmission de l'information sexuelle, on est en droit de se demander si une lésion partielle endommageant ce faisceau n'éliminera pas le caractère sexuel de la stimulation. En effet, certains patients dont la lésion endommage les faisceaux antérolatéraux mais épargne les cordons postérieurs rapportent avoir la même sensation lors de l'éjaculation qu'au passage de l'urine.

\section{AUTRES BILANS}

Des tests plus sophistiqués, tels l'EMG des sphincters et des muscles périnéaux et les potentiels évoqués péniens peuvent sonder l'état plus précis de la lésion médullaire en confirmant la présence ou l'absence d'activité musculaire ou sphinctérienne réflexe, signe d'un maintien du circuit sacré de l'érection. De même, le bilan urodynamique peut donner de précieuses informations sur l'état fonctionnel de l'innervation génitale, la présence d'activité détrusorienne et sphinctérienne étant signes d'innervation sacrée (parasympathique et somatique respectivement) alors que la présence d'hypertonie du col étant signe d'activité dorso-lombaire (sympathique). La manométrie anorectale confirme par ailleurs la présence d'activité sphinctérienne anale et permet d'en estimer la force.

\section{INTERROGATION CLINIQUE}

\section{Fonction sexuelle}

L'interrogatoire clinique, illustré au tableau 2 , est toujours utilisée comme source d'information sur la capacité sexuelle du sujet. Il comprend des questions relatives à l'érection réflexe qui peut survenir à la suite de la masturbation, des attouchements mutuels, ou de la pénétration. L'apparition de ces érections reflète un maintien de l'innervation sacrée malgré la lésion médullaire. Les questions relatives aux érections psychogènes sondent la voie dorso-lombaire, et plus précisément l'état de connexions entre les centres supérieurs et la voie dorso-lombaire.

L'interrogatoire clinique comporte également des questions sur l'érection spontanée et l'érection nocturne. L'érection spontanée sonde principalement la voie sacrée puisqu'elle apparait hors contexte sexuel mais en réponse à une stimulation directe (ex: toilette génitale) ou indirecte (contraction, distension vésicale) de l'appareil génital. L'érection nocturne sonde également la voie sacrée-réflexe puisque nos données préliminaires montrent qu'elle ne survient que chez les sujets dont la lésion préserve l'activité réflexe diurne [2].

Par ailleurs, la dysréflexie, diurne ou nocturne, sans causes précises est signe d'atteinte au-dessus de la double innerva- 
Tableau 2 : Interrogatoire clinique - Le patient a-t-il déjà noté les signes suivants?

DES ERECTIONS PAR STIMULATION

GENITALE

Pendant la masturbation

Pendant les attouchements

Pendant la pénétration

DES ERECTIONS PAR STIMULATION

PSYCHOGENES

Par les moyens visuels

Par des moyens auditifs

Par fantasme

Par désir du partenaire

DES ERECTIONS SPONTANEES

Lors d'un sondage urinaire

Lors d'un examen clinique

Au cours de la nuit

Au réveil

\section{DES EMISSIONS}

Spontanées

Suite à une stimulation psychogène

Suite à une stimulation génitale

DE LA DYSREFLEXIE

Sans cause précise

La nuit

tion génitale, tel que mentionné dans la section capacité éjaculatoire, alors qu'une émission spontanée décrite par le patient comme un écoulement visqueux ou une urine trouble peut être signe d'une lésion du cône médullaire.

Toutes ces questions à contenu sexuel sont donc importantes comme indice clinique et pour sonder le vécu du sujet. Cependant, elles ne devraient pas être l'unique source d'information sur la capacité sexuelle résiduelle des patients. En effet, nos études $[3,4]$ ainsi que celles d'autres chercheurs [11], montrent que les blessés médullaires ne sont pas toujours conscients de leur capacité sexuelle. L'interrogatoire clinique peut donc nous informer sur d'autres aspects, somatiques et viscéraux, qui peuvent être interprétés comme indice de fonctionnement du circuit sacré et/ou dorso-lombaire.

\section{Fonction somatique}

Lorsque l'examen complet n'est pas possible ou lorsque l'évaluation sexologique est effectuée par d'autres professionnels que le médecin, l'interrogatoire peut comporter des questions sur l'état de la sensibilité du patient en suivant la topographie des dermatomes. Des questions sur la fonction anorectale peuvent également révéler la présence d'activité réflexe périnéale. En effet, les blessés médullaires doivent effectuer des touchers rectaux quotidiens pour assurer l'évacuation des selles, si bien qu'ils peuvent avoir noté la présence de contractions sphinctériennes pendant le toucher ou la présence de béance anale. Lorsqu'il y a suivi, ces informations peuvent être vérifiées à domicile par le patient qui en ramènera les résultats lors de la prochaine entrevue. De la même façon, le réflexe bulbocaverneux peut être instruit au patient qui l'effectuera à domicile et en ramènera le résultat.

\section{Fonction urinaire}

Sur le plan urinaire, divers types de vessies neurogènes peuvent apparaitre, si bien que le mode d'évacuation nous donne des indices sur le type de lésion. Une lésion au dessus de la double innervation génitale préserve l'activité réflexe détrusorienne qui assure la miction- et l'activité sphinctérienne - qui assure la continence. Ces individus sont normalement soumis à un programme de cathétérismes intermittents, rarement accompagnés de fuites. Les individus souffrant d'une spasticité extrême peuvent néanmoins avoir subi une sphinctérotomie et être devenus porteurs de condoms urinaires. Par ailleurs, en cas de lésions complètes, ces individus ne sentiront pas le besoin d'uriner, qu'il s'agisse des premiers besoins (B1, B2), transmis par l'innervation sacrée, ou des besoins urgents et impérieux (B3, B4), transmis par l'innervation dorsolombaire.

Les patients dont la lésion endommage le cône médullaire perdent la contraction 
réflexe détrusorienne et souffrent d'hypotonie sphinctérienne. Néanmoins, ces lésions limitées au cône médullaire sont associées à une hypertonie du col, signe d'une activité sympathique dorso-lombaire. Ces patients suivent normalement un programme de cathétérismes intermittents qui peuvent être accompagnés de fuites à l'effort. Egalement, la sensation des besoins urgents ou impérieux peut révèler la présence d'activité dorso-lombaire.

Les patients aux lésions de la queue de cheval peuvent perdre l'innervation sacrée et dorso-lombaire auquel cas aucune contraction vésicale et une hypotonie du col et du sphincter seront enregistrées. Ces patients montreront des fuites continuelles et seront porteurs de condoms urinaires.

\section{ENREGISTREMENT DES REPONSES PENIENNES}

Tel que mentionné plus haut, l'interrogatoire clinique devrait être suivi d'un enregistrement objectif de la réponse sexuelle en situation réflexe et psychogène pour éviter la sous-estimation subjective du patient. La situation réflexe peut utiliser la masturbation, qui reste la meilleure source de stimulation directe, alors qu'un film érotique est parfaitement adapté au besoin de la stimulation psychogène $[3,4]$.

Les enregistrements peuvent être effectués à l'aide de jauges péniennes, reliées à un polygraphe ou un ordinateur, et qui mesurent les changements de circonférence pénienne durant l'érection. L'avantage de ces enregistrements est qu'il permettent une certaine intimité durant l'expérience sexuelle plutôt qu'une évaluation in situ du médecin traitant.

Idéalement, chaque séance de stimulation devrait être effectuée un jour différent, mais la réalité clinique nous oblige parfois à n'utiliser qu'une seule séance. Il est alors préférable de débuter avec la stimulation réflexe puisque le blessé médullaire n'a pas (ou peu) de sensation génitale. Ainsi, l'effet additif de la stimulation réflexe sur la stimulation psychogène sera limitée par le manque de sensation.

\section{DISCUSSION}

L'examen sexologique du blessé médullaire met en évidence l'état de l'innervation de l'appareil génital. De cet état, différents cas de figures, décrits précédemment, peuvent émerger ce qui nous permet d'émettre un diagnostic et pronostic de la fonction érectile et éjaculatoire de l'homme blessé médullaire. Ainsi, un patient qui présente des réflexes périnéaux, de l'activité détrusorienne et sphinctérienne présente des signes d'activité du circuit sacré. Lorsque cette activité est combinée à une anesthésie (ou hypoesthésie) sus-pubienne ainsi qu'à une absence de sensation du besoin d'uriner, la lésion est classifiée au-dessus de la double innervation de l'appareil génital. Lorsque l'activité sacrée est combinée à une anesthésie (ou hypoesthésie) inguinale, et au maintien de la sensation du besoin d'uriner urgent et/ou impérieux, le patient reçoit un diagnostic de lésion entre les deux systèmes.

Inversement, si un patient montre une absence de réflexes, possiblement accompagnée de béance anale, la lésion entrave le circuit sacré. Elle sera alors classifiée comme atteinte du cône médullaire si l'anésthésie (ou hypoesthésie) se limite à la région anale et péri-anale et sera classifiée comme atteinte de la queue de cheval si l'anesthésie englobe la face antérieure et postérieure des jambes. Qui plus est l'atteinte sera classée lésion haute de la queue de cheval si l'anesthésie englobe le pubis et parfois le nombril, alors qu'elle sera classée lésion basse si l'anésthésie se limite aux membres inférieurs. Dans le premier cas, l'atteinte risque d'entrainer une perte complète de la capacité érectile et éjaculatoire en entravant le fonctionnement du circuit sacré et dorso-lombaire.

Le bilan sexologique nous permet donc de mettre en évidence les particularités 
sexuelles du blessé médullaire et d'émettre, le cas échéant, un diagnostic différentiel entre une dysfonction strictement organique chez les blessés médullaires ou une dysfonction fortement psychogène. L'importance de cette distinction n'est plus à démontrer en sexologie traditionnelle et permet de mieux diriger le patient vers une approche médicale ou psychothérapeutique. L'importance du bilan apparait également dans la distinction entre le niveau vertébral et lésionnel. En effet, une paraplégie L1, en soi, ne signifie rien puisqu'elle peut représenter un niveau lésionnel, auquel cas on aura affaire à une lésion entre les deux systèmes permettant l'activité réflexe et psychogène. S'il s'agit d'une fracture en L1 et que l'éclatement de la vertèbre n'a endommagé que le cône médullaire, l'individu maintiendra une activité érectile et éjaculatoire psychogène mais non réflexe. Si l'éclatement de la vertèbre a endommagé le haut de la queue de cheval (en plus du cône médullaire probablement) il s'agira d'une lésion étendue de la queue de cheval avec peu d'activité résiduelle tant au niveau réflexe que psychogène.

L'évaluation de la fonction sexuelle du blessé médullaire présente donc une approche nouvelle qui documentera mieux le clinicien et le patient sur la capacité résiduelle de ce dernier et sur les traitements mieux adaptés au besoin de la clientèle.

\section{REFERENCES}

1. BODNER, D.R., LEFFLER, B. \& FROST, F. : The role of intracavernous injection of vasoactive medication for the restoration of erection in spinal cord injured males: a three year follow up. Paraplegia, 1992 : 118-120.

2. COURTOIS, F., CHARVIER, K., ROY, B \& LERICHE, A. : L'érection nocturne chez l'homme paraplégique: Des diagnostics erronés d'impuissance. 62ème Congrès ACFAS. Annales de l'ACFAS, $1994: 376$.

3. COURTOIS, F.J., CHARVIER, K.F., LERICHE, A. \& RAYMOND, D.P. : Sexual function in spinal cord injury men. I. Assessing sexual capability. Paraplegia, 1993 : 771-784.
4. COURTOIS, F.J., CHARVIER, K.F., LERICHE, A. \& RAYMOND, D.P. : Sexual function in spinal cord injury men. II. The quality of the response. Paraplegia, soumis.

5. DEROUET, H., GIRARDOT, P., CASPARI, D ET AL. : Acceptabilité de l'autoinjection dans le traitement de l'impuissance. Résultats à long terme. 84ème Congrès Français d'Urologie, Paris, 1990.

6. GIULIANO, F., RAMPIN, O., BENOIT, G. ET AL. : Commandes nerveuses périphériques de l'érection. Andrologie, 1993, 123-127.

7. GOLJI, H. : Experience with penile prosthesis in spinal cord injury patients. J. Urol., 1979 : 288-289.

8. HELLER, L., KEREN, O., ALONI, R. ET AL. : An open trial of vacuum penile tumescence : constriction therapy for neurological impotence. Paraplegia, $1992: 550-553$.

9. IWATSUBO, E., TANAKA, M., TAKAHASHI, K ET AL. : Non-inflatable penile prothesis for the managment of urinary incontinence and sexual disability of patients with spinal cord injury. Paraplegia, $1986: 307-310$.

10. KENDALL, H.O., KENDALL, F.P. \& WADWORHT, G.R. : Les muscles. Bilan et étude fonctionnelle, 1877, Baltimore : The Williams and Wilkins Company.

11. KENNEDY, S. \& OVER, R. : Psychophysiological assessment of male sexual arousal following spinal cord injury. Arch Sax Behav, 1990: 15-27.

12. LEE, L.M., STEVENSON, W.D. \& SZASZ, G. : Prostaglandin E1 versus phentolamine-papaverine for the treatment of erectile impotence: a double blind comparison. J. Urol, $1989: 549-550$.

13. NADIG, P.W., WARE, F.C. \& BLUMOFF, R. : Noninvasive device to produce and maintain an erection-like state. Urology, 1986 : 126-131.

14. NETTER, F.H. : The nervous system. New York: 1972, CIBA pharmaceutical Compagny.

15. ZASLER, N.D. \& IKATZ P.G. : Synergist erection system in the managment of impotence secondary to spinal cord injury. Arch Phys Med Rehabil, 1989 : 712-716.

\section{ABSTRACT}

Sexual function and sexological evaluation of paraplegic and tetraplegic

\author{
F. Courtois*, K. Charvier**, A. Leriche**
}

* Institut de réadaptation de Montréal, Montréal H3S 2J4 Canada ; ** Hôpital Henry Gabriel, 69230 Lyon 
This paper describes the sexological examination of spinal cord injured men. The complete exam includes a clinical interview, neurological examination, an EMG of the pelvic floor, a urodynamic examination and ano-rectal exploration, and the objective recording of the man's sexual response to reflex and psychogenic professionals or psychologists and sextherapists can proceed with a more thorough interview which evaluates number of the elements of the physical examination. The results of the investigation give rise to a number of clinical entities described in terms of lesion levels with respect to the innervation of the reproductive system. Lesions can therefore be classified as being located above the two pathways of erection, or extended to the cauda equina. Each lesion type is associated with a given pronostic of erectile and ejaculatory function. The accompanying physiological recording of penile responses further helps to establish a differential diagnosis between an exclusively organic dysfunction or a primarily psychogenic dysfunction in spinal cord injured men.

Key words : erection, ejaculation, spinal cord injury, paraplegia. 